unexpectedly announced in June that they might have trouble paying their dues in the coming year. Mattaj declined to name which nations were struggling.

"The situation is not easy for anyone," says Franco Bonacina, a spokesperson for the European Space Agency in Paris. As a result of the downturn, the agency has decided to put its plans for new missions largely on hold. Fresh projects are normally agreed during triennial meetings of research ministers, but there will be no meeting in 2011. Instead, the agency hopes that a lower-key delegation can approve two critical starts - an extension to the International Space Station programme until 2020, and funding to develop the next generation of the Ariane 5 rocket.

\section{A stimulating solution}

Not all plans for the future are on hold, however. Budget cuts have "never been something that's been asked of us," says Colin Carlile, director of the European Spallation Source, a new neutron-scattering facility to be located in Lund, Sweden. Carlile says that the Scandinavian member We states who origi"We are all very nally backed the worried about Lund site have so far the financial remained commitsituation." ted, as have other partners, including Spain. At present, the $€ 1.5$-billion facility is still on track to begin construction in 2013, he says, noting that the distant start date has insulated his group somewhat from the current financial problems.

Tim de Zeeuw, director-general of the European Southern Observatory, headquartered in Garching, Germany, says that in the next financial year he plans to ask for funds to start developing the European Extremely Large Telescope, a 42-metre-diameter behemoth. De Zeeuw points out that much of the telescope's $€ 1$-billion budget will go to hightech companies in Europe, which will design its optics and instrumentation. "If we want to stimulate our economy, this is a fine way of doing it," he says.

Womersley says that Britain hopes to participate in the new telescope, although the fiscal realities the country now faces may prove to be an obstacle. More generally, he hopes that Europe's patchwork of international partnerships will be able to negotiate the crisis. "We certainly don't want to see long-term damage to the future prospects of these organizations," he says.

Geoff Brumfiel

\title{
Sugar synthesis speeds up
}

Robot sub probes

Deepwater Horizon's underwater impact.

go.nature.com/6Q1gVR

\section{BOSTON, MASSACHUSETTS}

When biologists need a particular peptide or strand of DNA, they ask a machine to make it for them. Automation gives fast, inexpensive access to these molecules, and it has helped turbocharge biology.

But constructing complex carbohydrates from sugar building blocks still needs expert chemists, with researchers spending weeks - and tens of thousands of dollars forging molecules by hand.

Two groups of chemists now say they have independently developed automated carbohydrate synthesizers with the aim of making the molecules on demand. One machine is already churning out carbohydrates for clients, and the other will be available for labs to buy next year. Advocates say the machines could unplug a major bottleneck in the field of glycobiology, which studies the sugar chains (glycans) present on many proteins and lipids.

The main problem with carbohydrate synthesis is that sugar building blocks attach to each other in myriad ways to create a dazzling variety of branched structures — in contrast to peptides, which are made from a linear chain of amino acids linked by identical chemical bonds. Making the right carbohydrate means avoiding all the unwanted possibilities. great to manufacture. So Seeberger has spent the past decade completely remodelling his machine - overhauling the chemistry that links the sugars to the resin, for example.

Commercial machines will be available next year, he says. The device is being tested at Leiden University in the Netherlands and the University of Alberta in Edmonton, Canada. It is also being used by Ancora Pharmaceuticals, based in Medford, Massachusetts, a company co-founded by Seeberger to design and test carbohydratebased vaccines. Seeberger says that $90 \%$ of known mammalian carbohydrates can be constructed using just 35 building blocks, which he plans to supply in bulk.

Seeberger presented the early fruits of research using his machines at this week's American Chemical Society meeting in Boston, Massachusetts. For example, he is imaging sugar-covered bacterial cells - and targeting drugs at them - by attaching a variety of glycans to nanoparticles.

Not everyone thinks Seeberger's technology is currently versatile enough to make a wide range of carbohydrates on demand. "I don't think the chemistry is sufficiently well developed," says GeertJan Boons at the University of Georgia in Athens. But it could build libraries of carbohydrates using well-established
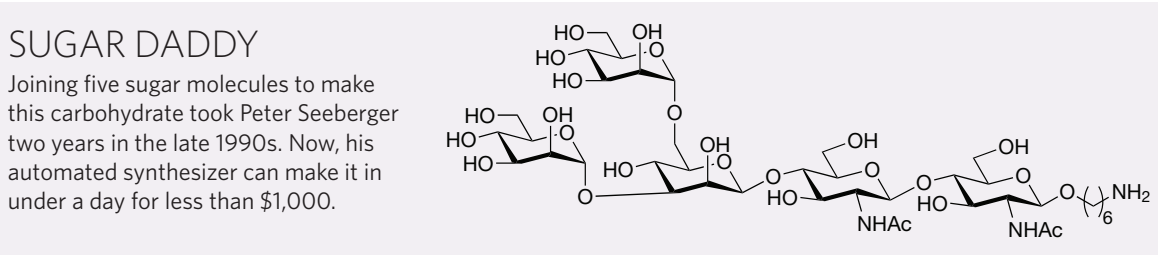

In 2001, chemist Peter Seeberger, who now directs the Max Planck Institute of Colloids and Interfaces in Potsdam, Germany, published the outline of a carbohydrate synthesizer that attached sugars one by one to a resin-linked chain (O. J. Plante, E. R. Palmacci and P. H. Seeberger Science 291, 1523-1527; 2001). The idea was based on solid-phase peptide synthesis, which won biochemist Bruce Merrifield the Nobel Prize in Chemistry in 1984; the resin holds on to the growing peptide or carbohydrate chain, with unreacted building blocks being washed away. But many researchers believed the chemistry behind Seeberger's proposal would be too complicated to be reliable, and the number of different sugars needed too synthetic routes, he adds.

Meanwhile, a complementary system is already delivering shorter carbohydrates than those targeted by Seeberger. Developed by Nicola Pohl of Iowa State University in Ames, it uses a hydrophobic fluorocarbon tag, rather than a resin, to anchor growing sugar chains in solution. Pohl's company, LuCella Biosciences in Ames, has been filling orders to build carbohydrates since November 2009. Pohl hopes that automated machines will persuade more biologists to study glycans rather than being deterred by their complexity. "A lot of our early work is about educating the biologists that these carbohydrates are now readily available," she says.

Richard Van Noorden 